\title{
Primary Breast Lymphoma: Exceptional Lesion with Particular Management
}

\author{
Olfa El Amine El Hadj ${ }^{1 *}$, Azza Gabsi², Aida Goucha1, Meyssa Belghith ${ }^{1}$, Khaled Rahal $^{3}$ and Amor Gamoudi \\ ${ }^{1}$ Department of Pathology, Carcinological Institute Salah Azaiez, Tunisia \\ ${ }^{2}$ Department of Medicine, Carcinological Institute Salah Azaiez, Tunisia \\ ${ }^{3}$ Surgical Department, Surgical Department Salah Azaiez, Tunisia
}

\begin{abstract}
Introduction: Non-Hodgkin's primary lymphoma of the breast is a rare entity. These tumors represent $0.04 \%$ to $0.52 \%$ of malignant breast pathology, $2.2 \%$ of extranodal lymphoma and $0.4 \%$ of all non-Hodgkin lymphoma. Clinical and radiological aspects show no special characteristics. The diagnosis is based on the presence of a lymphomatous proliferation strongly associated to the breast tissue and the diagnosis of an extra-mammary lymphoma must be removed except the presence of ipsilateral axillary lymphadenopathy.

Patients and methods: This is a retrospective study of 9 patients treated for non-Hodgkin's primary lymphoma of the breast. They were collected over a period of 14 years (2000-2013). They interested 1 case of follicular lymphoma, 1 case of large T-cell lymphoma and 7 cases of large B-cell lymphoma. The average age was 50 years, ranging from 30 to 76 years. The sex ratio was 0.11 ( 1 man/8 women). The median follow-up was 43 months (4-192). Six patients were older than 60 years. All the tumors were architecturally diffuse. The overall survival was $100 \%$ at one year $60 \%$ at 3 years. Seven patients achieved a complete remission after initial treatment; one had relapsed after 2 months. Among the 7 cases of large B cell lymphoma, two cases result from follicular lymphoma's transformation. The average processing time was 17 months.
\end{abstract}

Conclusion: The primary breast lymphoma is often diagnosed late. Treatment should be rapidly implemented. Indeed, the 5-year survival of stage I of the Ann Arbor classification is better than for stage II.

Keywords: Breast; Non-Hodgkin lymphoma; Histology; Immunohistochemistry

\section{Introduction}

Primary breast lymphoma (PBL) is defined as the affection of one or both breasts. Lymphomatous proliferation should be strongly associated to the breast tissue and the diagnosis of an extra-mammary lymphoma must be removed except the presence of ipsilateral axillary lymphadenopathy. Wiseman and Liao first defined PBL according to four criteria [1] the close anatomic proximity of mammary and lymphomatous tissue, absence of a previous diagnosis of an extramammary lymphoma, lack of evidence of a disseminated disease (except for the ipsilateral axillary nodal involvement) and an adequate quality of the histopathological specimen. These criteria are still widely accepted. PBL represents only $0.04 \%$ to $0.52 \%$ of breast malignancies, $2.2 \%$ of extranodal lymphomas and $0.4 \%$ all non-Hodgkin's lymphoma $[2,3]$. We propose in this work to review clinico-pathological characteristics and therapeutic particularities of non-Hodgkin primary lymphoma of the breast.

\section{Patients and Methods}

This is a retrospective study of 9 patients treated for non-Hodgkin's primary lymphoma of the breast. They were collected over a period of 14 years (2000-2013). Data on patient characteristics, the management procedures and evolution were collected from medical records and information sheets. This study concerns patients with a pathologic diagnosis of breast lymphoma. An extra-mammary location has been eliminated in all cases.

\section{Results}

The average age of patients was 50 years, ranging between 30 to 76 years. The population analyzed showed a female predominance ( 8 female with only one male).
In our study, three patients had breast pathology history. One patient had a history of an intra-ductal carcinoma. Two patients had a history of breast primitive follicular lymphoma which degenerates on diffuse large B cell lymphoma after 2 years and 10 months of evolution. One patient had a family history of infiltrating ductal carcinoma reported in a paternal aunt and a cousin. Three patients were already postmenopausal for 10,15 and 21 years, while 5 patients were still in childbearing age. One of our patients was under hormonal contraception for seven years. One of our patients, aged 30 years old, was pregnant for five months at the moment of breast lymphoma diagnosis.

The PBL discovery circumstances were mammary masses, increasing the volume of the breast and skin inflammatory signs. This pathology has interested the right side in 3 patients and left side in 5 patients. It was bilateral, at diagnosis, in only one patient. The most frequent location was the left upper outer quadrant in 5 patients, and the super-outer quadrant right in one case.

The average clinical size was $6.4 \mathrm{~cm}$, up to a maximum of $15 \mathrm{~cm}$. It was more than $5 \mathrm{~cm}$ in 3 patients. The ipsilateral axillary lymph nodes

*Corresponding author: Olfa EL Amine EL Hadj, Department of Pathology, Salah Azaiez Institue Boulevard 9 Avril 1006 Tunis, Tunisia, Tel: 21695936648; E-mail olfaelamine@yahoo.fr

Received August 05, 2016; Accepted December 21, 2016; Published December 23, 2016

Citation: El Hadj OEA, Gabsi A, Goucha A, Belghith M, Rahal K, et al. (2016) Primary Breast Lymphoma: Exceptional Lesion with Particular Management. J Cancer Sci Ther 8: 294-297. doi: 10.4172/1948-5956.1000431

Copyright: (c) 2016 El Hadj OEA, et al. This is an open-access article distributed under the terms of the Creative Commons Attribution License, which permits unrestricted use, distribution, and reproduction in any medium, provided the original author and source are credited. 
Citation: El Hadj OEA, Gabsi A, Goucha A, Belghith M, Rahal K, et al. (2016) Primary Breast Lymphoma: Exceptional Lesion with Particular Management. J Cancer Sci Ther 8: 294-297. doi: 10.4172/1948-5956.1000431

\begin{tabular}{|c|c|c|c|}
\hline \multicolumn{1}{|c|}{} & \multicolumn{1}{|l|}{ Histological Type } \\
\hline 1 & Stage & Diffuse large B cell Lymphoma & Treatment \\
\hline 2 & I & Diffuse large B cell Lymphoma & Chemotherapy-Radiotherapy \\
\hline 3 & I & Diffuse large B cell Lymphoma & Chemotherapy-Radiotherapy \\
\hline 4 & I & Diffuse large B cell Lymphoma & Chemotherapy \\
\hline 5 & II & Diffuse large B cell Lymphoma & Chemotherapy \\
\hline 7 & II & Diffuse large B cell Lymphoma & Chemotherapy \\
\hline 8 & II & Diffuse large B cell Lymphoma \\
\hline
\end{tabular}

Table 1: details of therapeutic options received by the 9 patients included in the study.

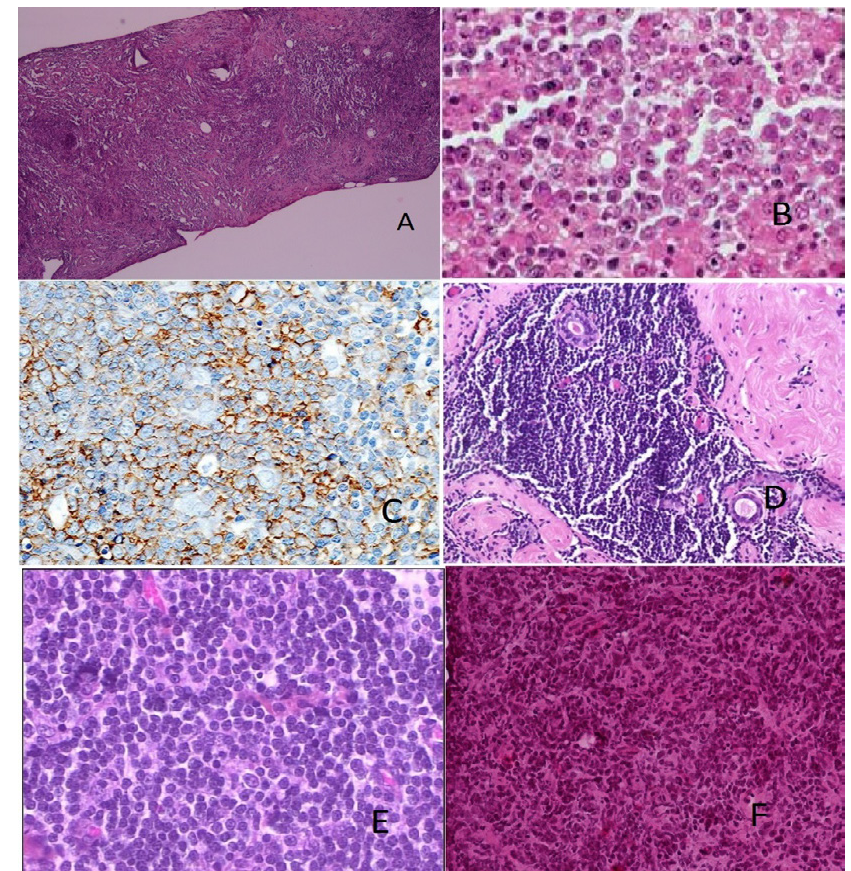

Figure 1: Diffuse lymphoma proliferation with nodular enhancement: different images observed in our patients A: (HEx40) Fine needle biopsy showing a diffuse B Cell lymphoma infiltrating breast parenchyma; B: (HEx200) Large tumor cells with prominent nucleoli: (Immunohistochemistryx200): tumor cells showing diffuse expression of CD 20 antibody; D (HEx100): DBCL infiltrating mammary parenchyma; E and F (HEx200): Atypical tumor cells with pleomorphic and hyperchromatic nuclei.

were palpated in 5 patients. In a patient with left axillary lymph nodes, clinical examination revealed a supraclavicular lymphadenopathy. The histological exam concluded to a reactional node in all cases.

A mammography was performed in 6 patients, always associated with ultrasound. The average tumor size in the mammography was $40.5 \mathrm{~mm}$, ranging from 10 to $70 \mathrm{~mm}$. Mammography showed different aspects: a poorly circumscribed opacity with indefinite and irregular contours or spiculated dense center, a dense heterogeneous and irregular mass with retraction and thickening of superficial skin plans.

Observed ultrasound aspects were: a hypoechoic irregular mass, a multilobulated mass or a rounded supra areolar opacity, heterogeneous hypoechoic, surrounded by a hyperechoic halo interrupting the glandular architecture and hypervascular on color Doppler. MRI was performed in only two patients and showed a spiculated lesion with polycyclic limits. This lesion is hypo signal T1, ISO signal T2 and hyper signal FS. The scan of the male patient showed contiguous right axillary lymph nodes of 2 to $4 \mathrm{~cm}$ in diameter, a large right parietal mass of 11 $\mathrm{cm}$ and a multinodular splenomegaly. In our study 5 patients had stage I at diagnosis and 4 patients had stage II (Table 1).

By histological exam, 7 cases correspond to a large B-cell lymphoma (LBCL) (Figure 1), a case of follicular lymphoma and one case of large T-cell lymphoma. Immunohistochemical study allowed us to confirm the diagnosis of non-Hodgkin's lymphoma. We used a large panel of antibody: B marker (CD20), T marker (CD3), CD30, CD45, CD15 and CD10. In cases of LBCL, the I.H.C study showed a diffuse and intense staining with anti-CD20 (Figure 1). In case of T cell lymphoma, the tumor cells expressed diffusely the anti CD3. In follicular lymphoma, we found a diffuse expression of anti CD 20 and CD 10. Details of immunohistochemical results are summarized in Table 2. Treatment consisted on surgery, chemotherapy and/or radiotherapy. Five patients had only chemotherapy; four had chemotherapy with radiotherapy. One patient had received radiotherapy alone (Table 1 ). The surgery was performed in 7 cases. Five patients had a lumpectomy with axillary dissection while 2 had a radical surgery mastectomy with axillary dissection type Patey. Progression free survival was $43 \%$ at 3 years and $24 \%$ at 5 years. The median follow-up of our patients was 41 months with extremes ranging from 1 to 159 months. Overall survival was $60 \%$ at 3 years and $42 \%$ at 5 years.

\section{Discussion}

The term "primary breast lymphoma" (PBL) is used to define a malignant lymphoma primarily occurring in the breast in the absence of previously detected lymphoma localizations. It occurs specially in women whose median age is between the fifth and sixth decade $[4,5]$. Only a few cases have been reported in males [6]. In this report 8 patients among 9 were females.

PBL is also a tumor of elderly women [2,3]. However, some authors have shown that a median of 28 years was found in $15 \%$ of cases in pregnant women or during breast-feeding [7]. In our study, a patient with DBCL was 30-year-old and was pregnant at the fifth month.

The association lymphoma and pregnancy is increasingly described and some authors have found that it is more important than pregnancycarcinoma association. Thus, we can conclude that hormonal disturbances likely play a role of inducing proliferation lymphoma [7].

The affection is often unilateral. In $18 \%$ of cases, it is bilateral; it can be simultaneous $(12 \%)$ or sequential $(6 \%)[8,9]$. The mode of revelation is almost always a breast tumor with an inflammatory mastitis [2]. The axillary lymph nodes are found in $20 \%$ to $40 \%$ of cases [10].

The primitive character of breast lymphoma is based on clinical investigation. Indeed, we should eliminate secondary breast's invasion during the natural evolution of non-Hodgkin lymphoma [11].

The radiologic imaging' aspects are nonspecific. Mammogram often shows a well limited mass with homogeneous density and benign 


\begin{tabular}{|c|c|c|c|c|c|c|c|}
\hline Patient & Histological Type & CD20 & CD45 & CD3 & CD30 & CD15 & CD10 \\
\hline 1 & Diffuse large B cell Lymphoma & + & + & - & - & - & - \\
\hline 2 & Diffuse large B cell Lymphoma & + & + & - & - & - & - \\
\hline 3 & Diffuse large B cell Lymphoma & + & + & - & - & - & - \\
\hline 4 & Diffuse large B cell Lymphoma & + & + & - & - & - & - \\
\hline 5 & Diffuse large B cell Lymphoma & + & + & - & - & - & - \\
\hline 6 & Diffuse large B cell Lymphoma & + & + & - & - & - & - \\
\hline 7 & Diffuse large B cell Lymphoma & + & + & - & - & - & - \\
\hline 8 & Follicular Lymphoma & + & + & - & - & - & + \\
\hline 9 & Large T cell Lymphoma & - & + & + & - & - & - \\
\hline
\end{tabular}

Table 2: Details of immunohistochemical profile of 9 primary breast lymphomas.

appearance, evoking a cyst, a fibroadenoma or phyllodes tumor. Less frequently, it is a mastitis appearance with diffuse increase breast density or an ill-defined or speculated contour mass [12]. Rarely, a suspicious aspect of malignancy is observed, but there is never stellar opacity or microcalcifications [10]. Ultrasound presentation is not specific, usually as a hypoechoic and homogenous mass, with regular contours. Rarely a mastitis appearance is seen on ultrasound. The discrepancy between alarming clinical presentation and reassuring mammography may suggest the diagnosis [10].

The standard histological examination left little doubt in case of medullary and anaplastic carcinoma whereas lymphoma's diagnosis is often difficult $[13,14]$. Usually, immunohistochemical study on paraffin sections confirms the diagnosis [15]. Diffuse large B-cell lymphoma is the most frequent subtype. It represents $45 \%$ to $80 \%$ of all breast lymphomas subtypes. All histological types have been described in breast. Lymphoma of the MALT type marginal zone are common ( $0 \%$ to $44 \%)$ and Burkitt's lymphoma often associated with bilateral involvement [16]. In our work, no cases of Burkitt's lymphoma has been described this can be related to a particular geographical distribution of this type of lymphoma $[13,14]$.

The management strategies for PBL vary broadly, from surgical intervention to combination chemotherapy and radiotherapy. However, there is no up to date standard guideline for PBL treatment. Mastectomy for PBL is not well-supported because it shows neither improved survival nor reduced risk of recurrence. Several studies have recommended that minimally invasive surgery is the preferable option because extensive surgery may carry a high risk of morbidity. Also, axillary dissection adds no therapeutic advantage [17]. At present, early stages of PBL can be successfully treated with combination therapy, in which $\mathrm{CHOP}$ regimen is the most common chemotherapeutic agent. Several investigators have recommended a treatment regimen involving combination chemotherapy with or without radiation therapy [17].

Ganjoo et al. [18] searched the Stanford lymphoma database for patients with documented lymphoma involving the breast between 1981 and 2005 and included 37 patients in their analysis. Their DLBCL patients received doxorubicin-based chemotherapy, with $70 \%$ receiving involved-field radiotherapy (IFRT) with doses of 36005040 cGy. A single patient additionally received cytotoxic intrathecal therapy. No recurrences occurred in the involved breast. Among the DLBCL patients, 5-year progression-free survival (PFS) was $61 \%$, with a median follow-up of 3.8 years (range: 5 months to 19 years) and a 5 -year OS was estimated at $82 \%$. Patients with indolent lymphoma had an estimated 5-year PFS of $76 \%$ and an OS of $92 \%$. The authors conclude that DLBCL of the breast can be successfully treated with doxorubicin-based chemotherapy alone or with IFRT.

In 2008, Ryan et al. [19] published the results of a retrospective international study of 204 eligible patients with PBL DLBCL histological subtype attending the International Extranodal Lymphoma.

Study Group-affiliated institutions from 1980 to 2003. The median age was 64 years, median OS was 8.0 years, and median progression-free survival (PFS) was 5.5 years. There was no benefit from mastectomy, as opposed to biopsy or lumpectomy. At a median follow-up time of 5.5 years, in $35 \%$ of patients the tumour had progressed: $16 \%$ locally in the same or contralateral breast, $5 \%$ in the CNS and $14 \%$ in other extranodal sites. According to the authors, the combination of limited surgery, anthracycline-containing chemotherapy, and involved-field $\mathrm{RT}$ produced the best outcome in the pre-rituximab era. In conclusion, they postulated that a combined therapy was the best method of treating patients with PBL. They suggested using prophylaxis to avoid central nervous system involvement. A similar opinion was expressed by Aviles et al. [20].

The prognosis of diffuse large B-cell lymphoma is poor. Among 215 cases whose outcome was known, Prevot [19] notes that more than half of the patients died within a period ranging from 4 days to 178 months after diagnosis. Baltali estimated survival rate at 5 -year $30 \%$ to 50\%. [20] Histological type and clinical stage of the disease are the two main prognostic factors. Indeed, the risk of death is nearly double if PBL stage II versus stage I [21-23].

\section{Conclusion}

Primary lymphoma is a very rare pathology. If symptoms are not yielding to treatment, histological sampling is imperative. Treatment should be quickly implemented. Indeed, the five-year survival for stage I of the classification of Ann Arbor is better than for stage II.

\section{References}

1. Wiseman C, Liao KT (1972) Primary lymphoma of the breast. Cancer 29: 17051712.

2. Boudhraa K, Amor H, Kchaou S, Ayadi A (2009) Primitive Breast Lymphoma: About a subclinical case. The Medical Tunisia. 78: 215-218.

3. Seker M, Bilici A, Ustaalioglu BO, Yilmaz B (2011) Clinicopathologic features of the nine patients with primary diffuse large $B$ cell lymphoma of the breast. Arch Gynecol Obstet. 284: 405-409.

4. Brustein B, Fllippa D, Kimmel MA, Lieberman PH (2009) Malignant lymphoma of the breast: A study of 53 patients. Ann Surg 205: 144-150.

5. Jain MF, Khandekar SL, Mahadani JW, Raut WK (2015) Report of a case of primary breast lymphoma highlighting the importance of fine needle aspiration cytology as an initial diagnostic tool. J Cytol 32: 127-129.

6. Freeman C, Berg J, Cutler SJ (1972) Occurrence and prognosis of extranoda lymphomas. Cancer 29: 252-260.

7. Jernestron P, STHER JM (1997) Primary lymphoma of the mammory gland JAMA 201: 503-506.

8. Sokolov T, Shimonov M, Blickstein D, Nobel M, Antebi E (2000) Primary lymphoma of the breast: Unusual presentation of breast cancer. Eur J Surg 166: 390-393. 
Citation: El Hadj OEA, Gabsi A, Goucha A, Belghith M, Rahal K, et al. (2016) Primary Breast Lymphoma: Exceptional Lesion with Particular Management. J Cancer Sci Ther 8: 294-297. doi: 10.4172/1948-5956.1000431

9. Duncan VE, Reddy VV, Jhala NC, Chhieng DC, Jhala DN (2006) Non-hodgkin's lymphoma of the breast: A review of 18 primary and secondary cases. Ann Diagn Pathol. 10: 144-148.

10. Fatnassi R, Bellara I (2005) Primary non-Hodgkin's lymphomas of the breast. Report of two cases. J Gynecol Obstet Biol Reprod (Paris) 34: 721-724.

11. Boffetta $P$ (2011) Epidemiology of adult non-hodgkin lymphoma. Ann Onco 22: 27-31.

12. Cohnen M, Saleh A, Germing U, Engelbrecht V, Modder U (2002) Imaging of supradiaphragmatic manifestations of extranodal non-Hodgkin's lymphoma. Radiologe 42: 960-969.

13. Jeanneret-Sozzi W, Taghian A, Epelbaum R, Poortmans PH (2008) Primary breast lymphoma: Patient profile, outcome and prognostic factors. A multicenter Rare Cancer Network study. BMC Cancer 8: 86.

14. Levine PH, Zamuco R, Yee HT (2004) Role of cytology cytology in breast lymphoma. Diagn Cytopathol 30 332-340.

15. Gaulard PH, et Thieblemont C (2008) Non-Hodgkin's lymphoma: Classification histopathology. French Society of Hematology (2ndedn).

16. Basing BA, Qing L, Rothman N, Zhang Y (2012) Current understanding of lifestyle and environmental factors and risk of non-hodgkin lymphoma: An epidemiological update. J Canc Epidemiol 27

17. Jabbour G, El-Mabrok G, Al-Thani HA, El-Menyar A, Al Hijji I, et al. (2016) Primary breast lymphoma in a woman: A case report and review of the literature. Am J Case Rep 17: 97-103.

18. Ganjoo K, Advani R, Mariappan MR, McMillan A, Horning S (2007) NonHodgkin lymphoma of the breast. Cancer 110: 25-30.

19. Gualco G, Bacchi CE (2008) B cell and T-cell lymphomas of the breast: Clinicalpathological features of 53 cases. Int Surg Pathol 16: 40.

20. Aviles A, Delgado S, Nambo MJ, Neri N, Murillo E, et al. (2005) Primary breast lymphoma: Results of a controlled clinical trial. Oncology 69: 256-260.

21. Prevot S, Hugol D, Tourneau TA, Audoini J, Diebold J (1997) Primary nonhodgkin's malignant lymphoma of the breast. Anatomopathologic diagnosis of 14 cases. Bull Cancer 77: 132-136.

22. Dhabhar BN (1992) Primary lymphoma of breast-report of six cases and review of literature. 17: 85-91.

23. Wong WW, Schild SE, HalyardMY, Schomberg PJ (2002) Primary non-Hodgkin lymphoma of the breast: the Mayo clinic experience. J Surg Oncol 80:19-25. 